Supporting Information for

\title{
Self-Assembly of Chiral Nematic-like Films with Chiral Nanorods Directed by Chiral Molecules
}

Tianwei Duan ${ }^{1 \dagger}$, Jing $\mathrm{Ai}^{2 \dagger}$, Yingying Duan ${ }^{2 *}$, Lu Han ${ }^{2 *}$ and Shunai Che ${ }^{1,2^{*}}$

${ }^{1}$ School of Chemistry and Chemical Engineering, State Key Laboratory of Composite Materials, Shanghai Key Laboratory for Molecular Engineering of Chiral Drugs, Shanghai Jiao Tong University, 800 Dongchuan Road, Shanghai, 200240, China.

${ }^{2}$ School of Chemical Science and Engineering, Tongji University, 1239 Siping Road, Shanghai, 200092, China.

These authors contributed equally to this work.

*Correspondence to: chesa@sjtu.edu.cn; luhan@tongji.edu.cn; yyduan@tongji.edu.cn. 


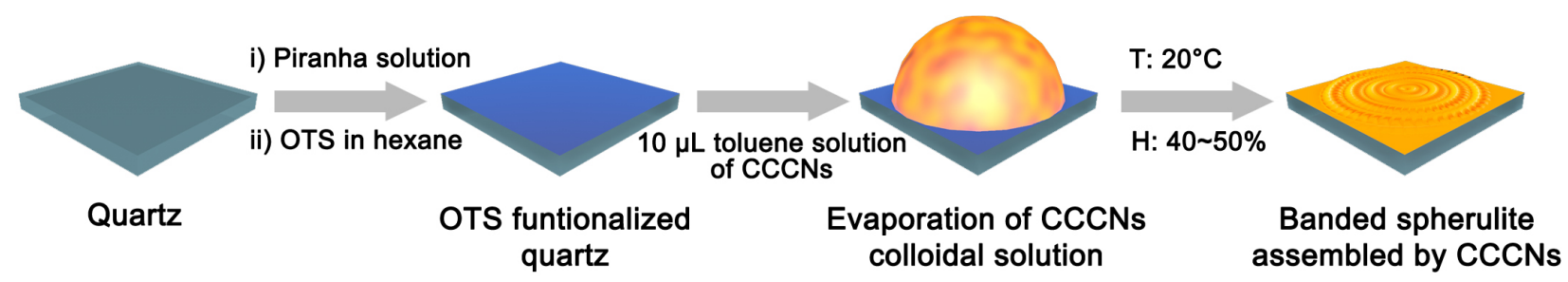

Scheme S1. Schematic drawing for self-assembly of CCCNs on quartz substrate. OTS: noctadecyltrichlorosilane; T: temperature; H: Humidity.

The fabrication procedure of CNFs was as follows:

(i) Before self-assembly of CCCNs, the quartz substrate was cleaned and modified.

(ii) The $0.5 \mathrm{~cm} \times 0.5 \mathrm{~cm}$ quartz substrate is cleaned by ultrasonic wave for three times with deionized water, and then three times with the mixed solution of acetone and ethanol with the volume ratio of 1:1.

(iii) In the modification step, the quartz substrate was treated with piranha solution $\left(\mathrm{VH}_{2} \mathrm{O}_{2}: \mathrm{VH}_{2} \mathrm{SO}_{4}=1: 3\right)$ to enrich hydroxyl groups on surface.

(iv) After that, the reaction was carried out in n-octadecyltrichlorosilane solution dissolved in hexane. Due to the existence of a small amount of water molecules, the siloxy groups can be slowly hydrolyzed and grafted on quartz substrate.

(v) Finally, the OTS modified quartz substrate was placed on a horizontal table, and CCCNs collidial solution (60 $100 \mathrm{mg} / \mathrm{mL}$ in toluene) was dripped on the substrate to form CNF with different thickness at room temperature of $\sim 20^{\circ} \mathrm{C}$. 

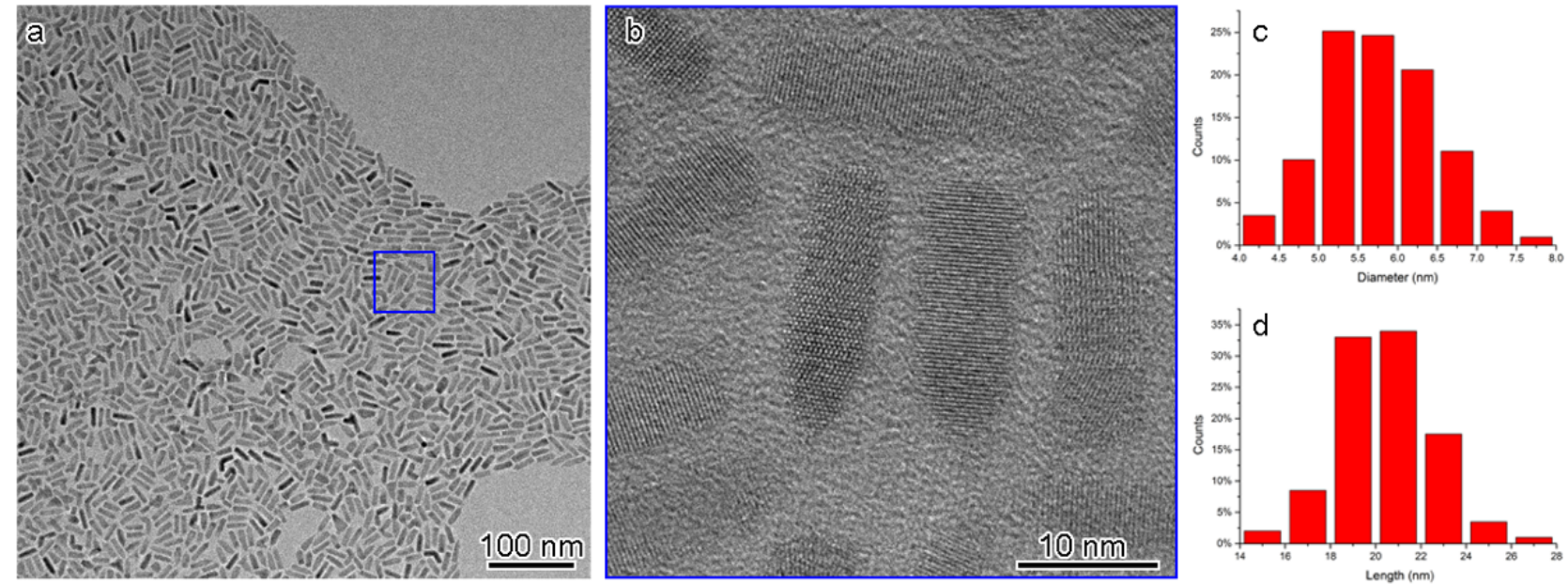

Figure S1. TEM images of CCCNs. (a) Low magnification TEM image of CCCNs. (b) High magnification TEM image of CCCNs. (c and d) Size distribution of the diameter and length of CCCNs from data statistic of 200 particles, respectively.

Majorities of nanoparticles showed rods morphology, and the minorities exhibited bent (bipod) and tripod morphology. The mean values for diameter and length form statistic were $5.8 \mathrm{~nm}$ and $20.2 \mathrm{~nm}$, respectively. 

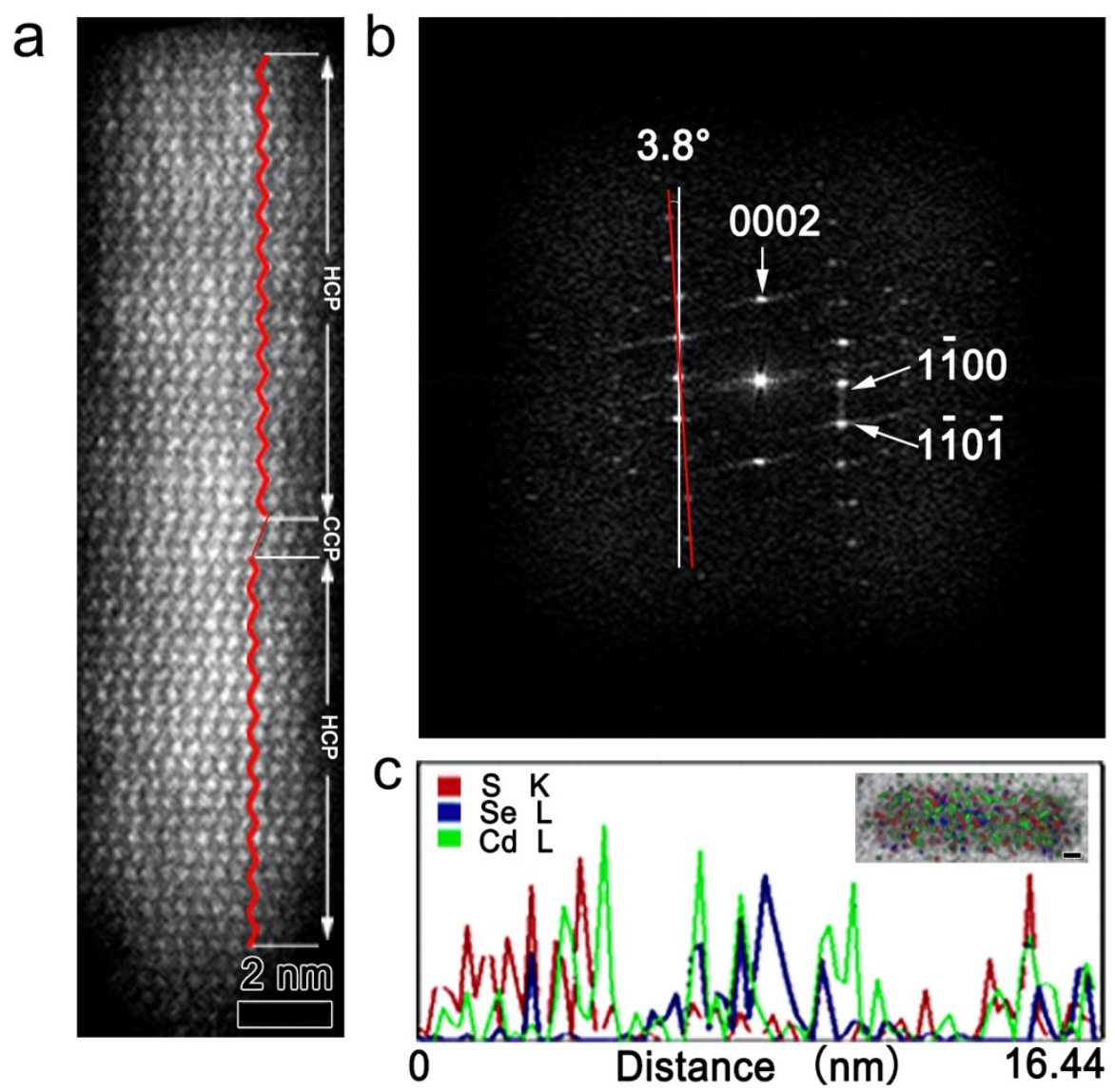

Figure S2. Crystal structure and composition of a single CCCN. (a and b) HAADF-STEM image and corresponding FDs of a single CCCN, respectively. (c) EDS mapping of a single nanorod. 


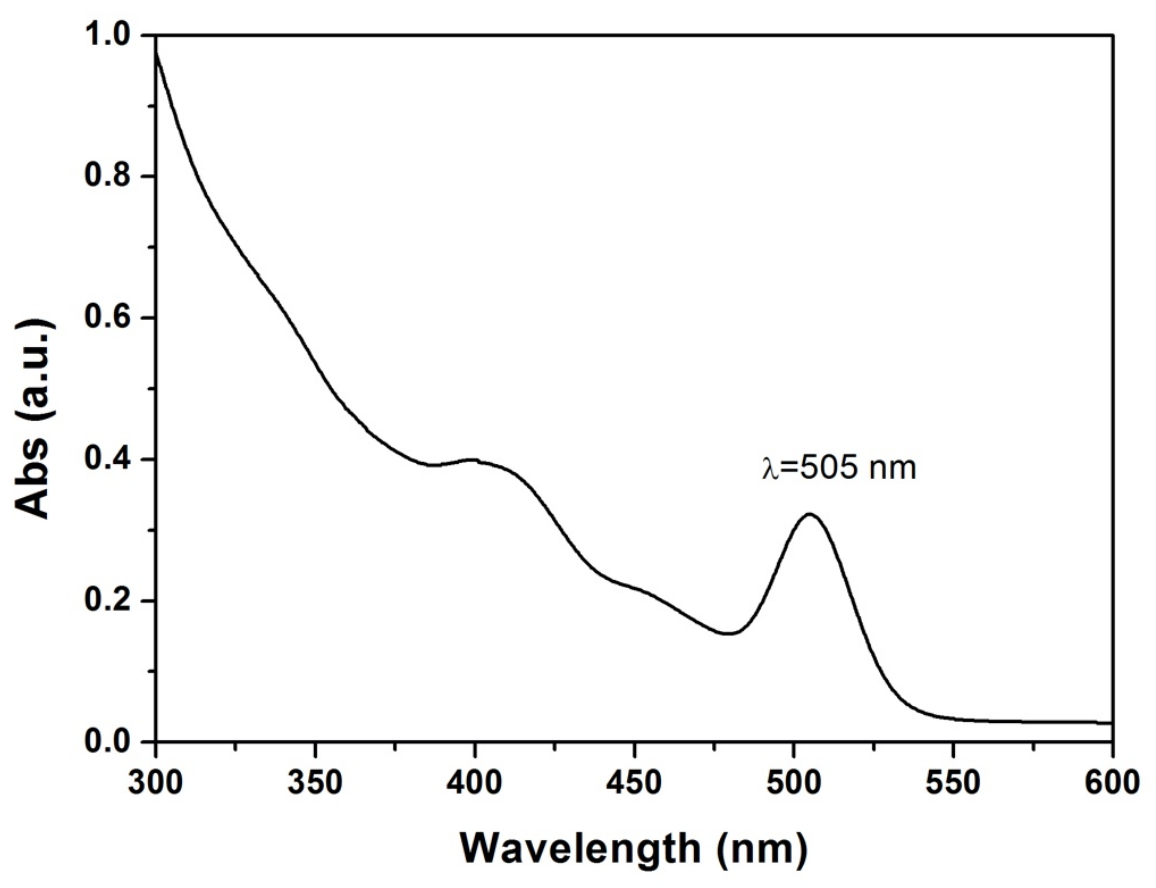

Figure S3. UV-Vis absorption spectra of CdSe cores.

For determination of size of CdSe cores used in synthesis of CCCNs, Peng's equation ${ }^{1}$ was used to calculate the diameter of CdSe.

$\mathrm{D}=\left(1.6122 \times 10^{-9}\right) \lambda^{4}-\left(2.6575 \times 10^{-6}\right) \lambda^{3}+\left(1.6242 \times 10^{-3}\right) \lambda^{2}-0.4277 \lambda+41.57$

In the above equation, $\mathrm{D}(\mathrm{nm})$ is the size of nanocrystals and $\lambda(\mathrm{nm})$ is the wavelength of the first excitonic absorption peak of corresponding sample. As for the CdSe cores used in experiment, $\lambda$ was $505 \mathrm{~nm}$ and D was $2.4 \mathrm{~nm}$. 


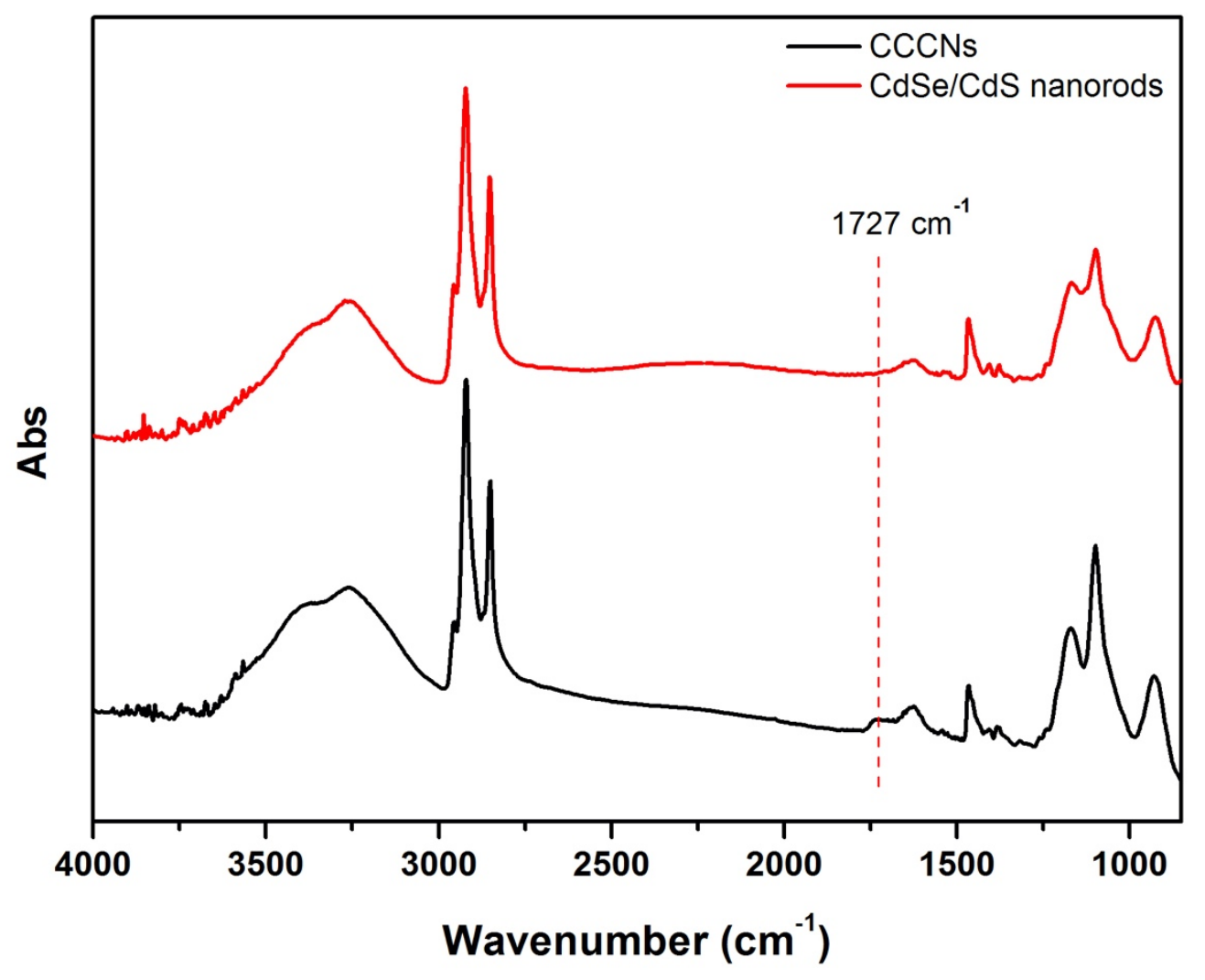

Figure S4. FTIR spectra of CCCNs and the CdSe/CdS nanorods synthesized without using cholic acid. 


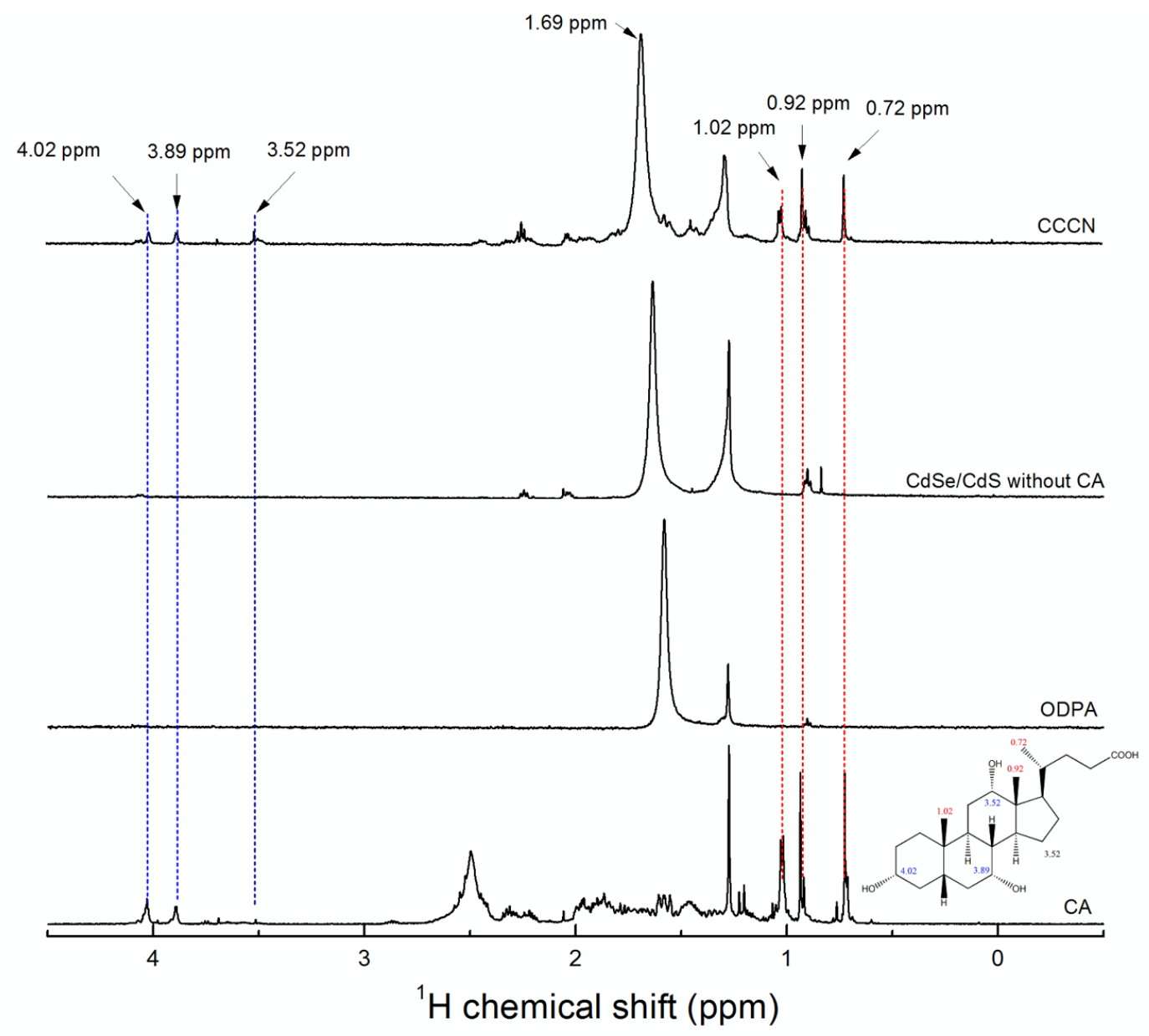

Figure S5. ${ }^{1} \mathrm{H}$ NMR of CCCNs, CdSe/CdS nanorods synthesized without CA, ODPA and CA

Figure S5 plots ${ }^{1} \mathrm{H}$ NMR spectra for CCCNs, $\mathrm{CdSe} / \mathrm{CdS}$ nanorod that synthesized without $\mathrm{CA}$ and CA molecules. As shown in Figure S5, the peaks centered at $\sim 4.02, \sim 3.89, \sim 3.52 \mathrm{ppm}$ are assigned to the $\alpha-\mathrm{H}$ of $-\mathrm{CH}$ - groups which connected to $-\mathrm{OH}$ of $\mathrm{CA}$ (marked as blue lines and blue number in the inset), and the peaks centered at $\sim 1.02, \sim 0.92$, $\sim 0.72 \mathrm{ppm}$ are referred to the $\mathrm{H}$ of $\mathrm{CH}_{3}$ - groups (marked as red lines and red number in the inset). These characteristic chemical shifts appear in the nanorods synthesized with CA, but not in the nanorods synthesized without CA, which proves that CA exists on the surface of nanorods 

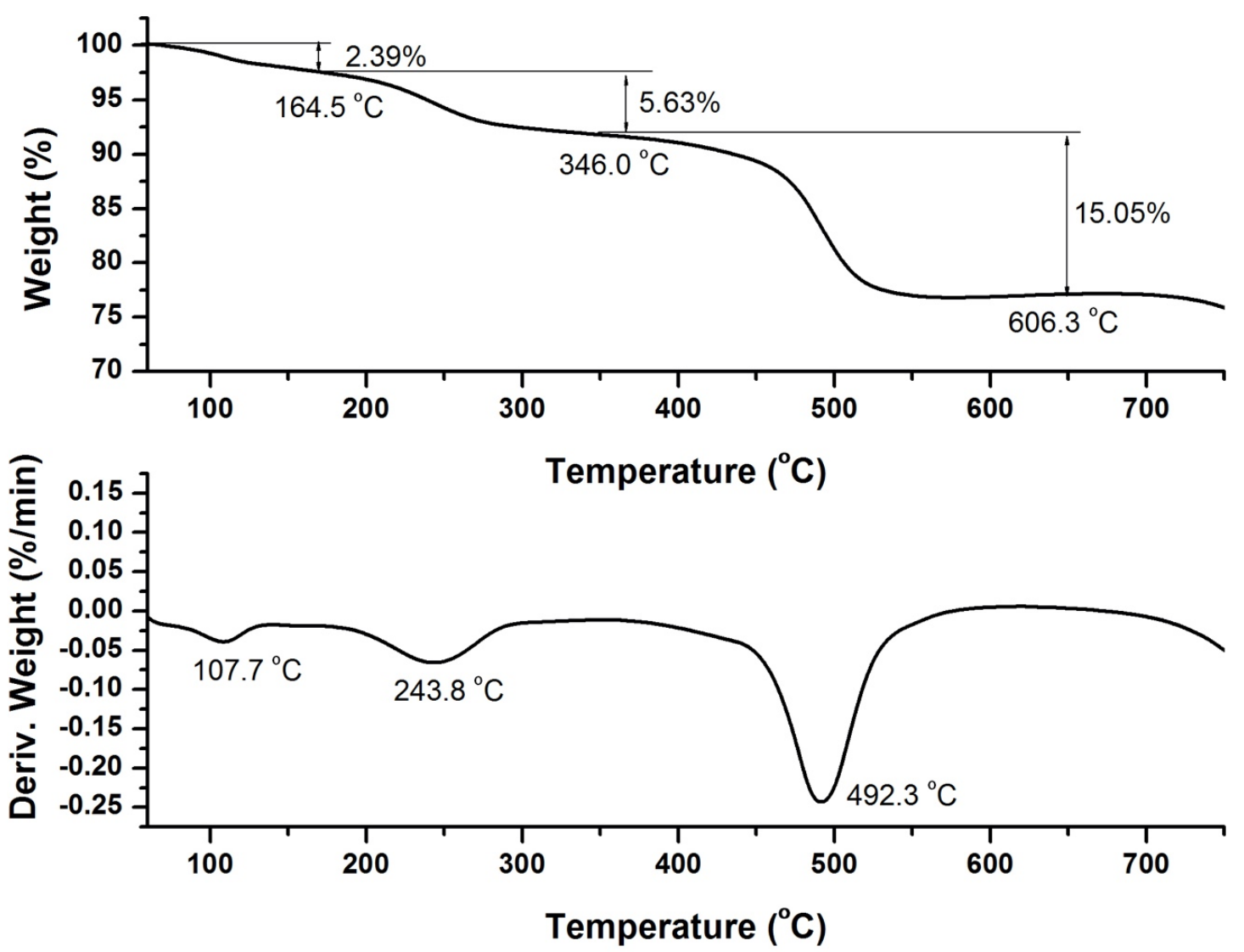

\section{Figure S6. TGA and DTA of CCCNs.}

The TGA curves presented in Figure S6 exhibit three mass loss regions:1) $50 \sim 150{ }^{\circ} \mathrm{C}$; 2) $150 \sim 300{ }^{\circ} \mathrm{C}$; 3) $300 \sim 500{ }^{\circ} \mathrm{C}$. The weight loss in the low temperature below $150{ }^{\circ} \mathrm{C}$ was related to the removal of absorbed water. The region $150 \sim 300{ }^{\circ} \mathrm{C}$ originates from the evaporation of neutral molecules like L-type ligands (such as TOP and TOPO), which have relatively weak attachment to the CCCNs surface. The region between $300 \sim 500{ }^{\circ} \mathrm{C}$ is derived from ionically bound ligands like X-type ligands, such as CA and ODPA, which have stronger attachments to the surface atoms on the CCCNs. The total organic and inorganic content was $23.07 \%$ and $76.93 \%$ by mass, respectively. The molar ratio of CA to ODPA is $\sim 0.53$, which can be determined from the ratio of peak area caused by a proton from characteristic chemical shift peaks (the peak centered at $\sim 4.02 \mathrm{ppm}$ from $-\mathrm{CH}$ - of $\mathrm{CA}$ and the peak centered at $\sim 1.69$ ppm from $-\mathrm{CH}_{2}$ - of ODPA in Figure S5). Thus, the weight ratio of CA to ODPA is 0.65 , which correspond to mass fraction of $6 \%$ in $\mathrm{CCCNs}$ (referred to $\mathrm{CA} /(\mathrm{CCCNs}+$ organic ligands)). 

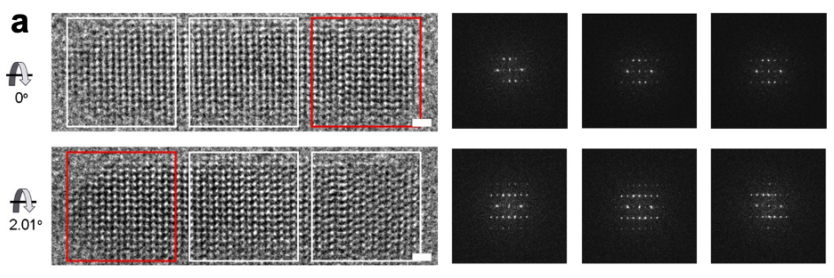

b
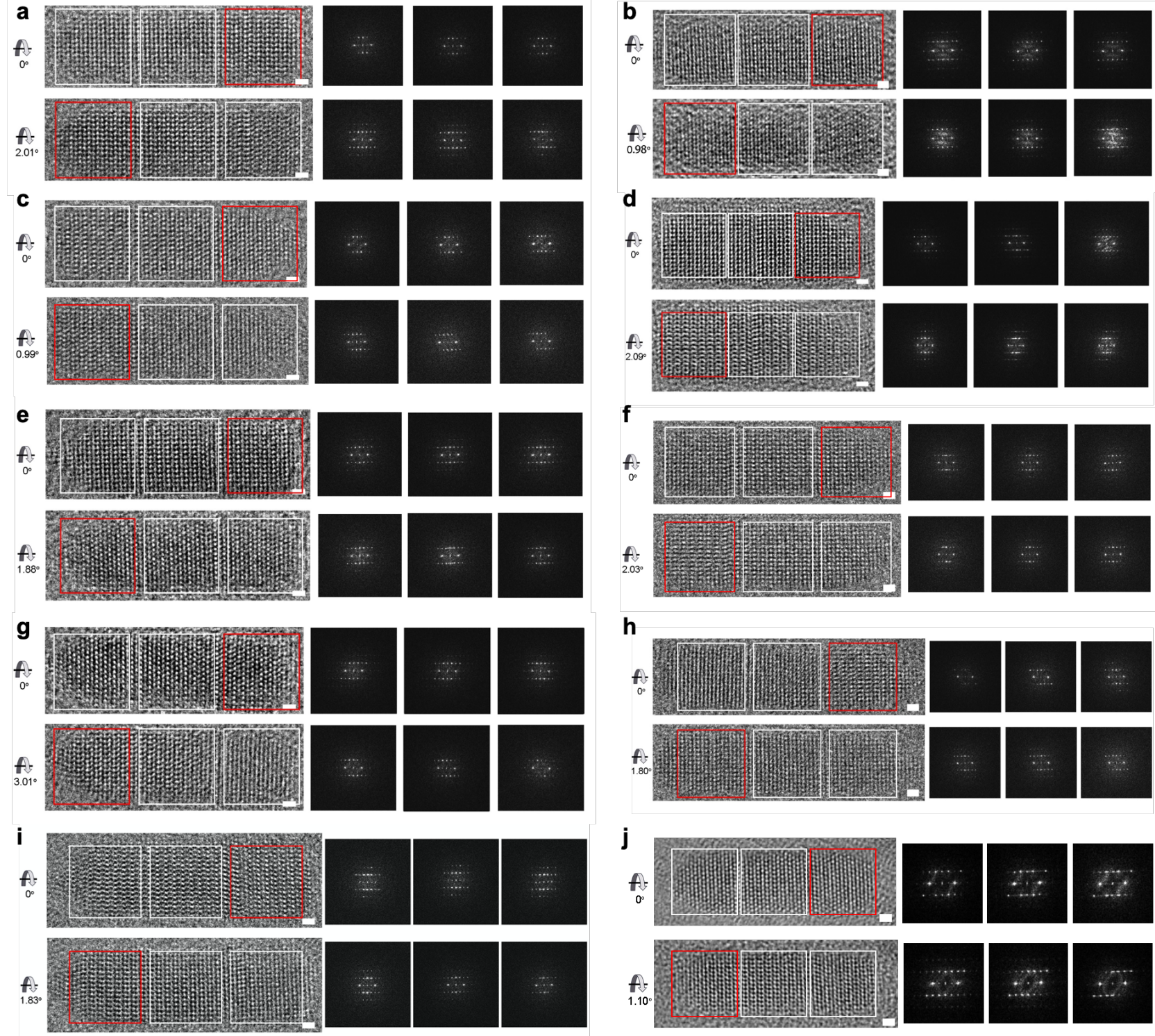

$\mathbf{f}$
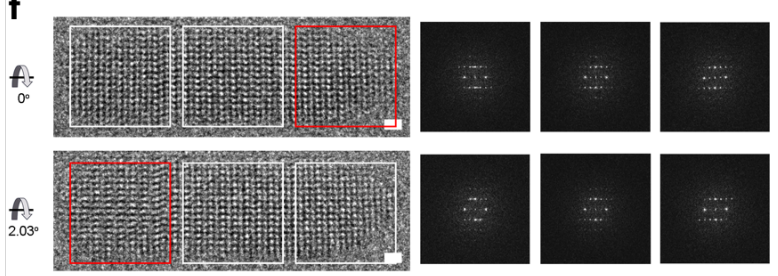

h
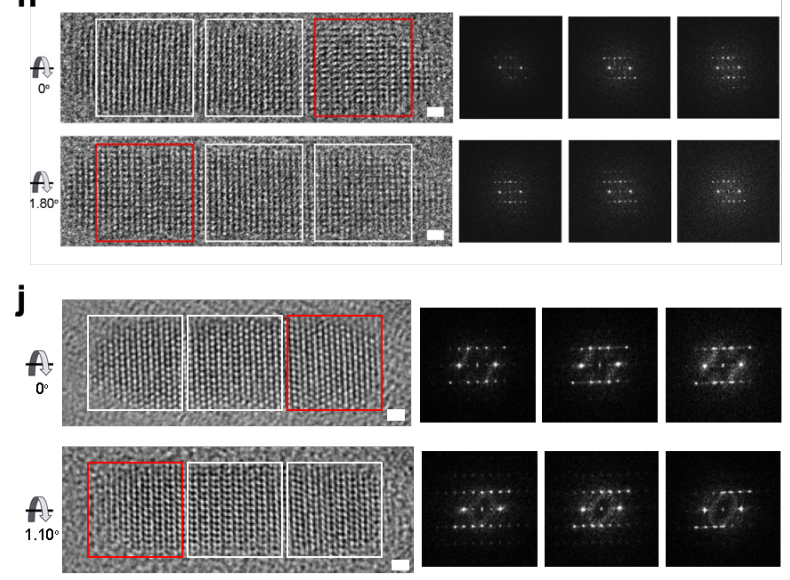

Figure S7. HRTEM images and the corresponding FDs of 10 right-handed CCCNs taken from different tilting angles along the axial direction of CCCNs.

The chiral nature of 10 CCCNs were characterized by high-resolution TEM (HRTEM) observations, as shown in

Figures S7. The nanorod showed the typical contrast of the close-packing structure along the $[110]_{\mathrm{cub}} /[11 \overline{2} 0]_{\mathrm{hex}}$ direction, and the axial direction was determined to be $[111]_{\mathrm{cub}} /[0001]_{\text {hex }}$. FDs were taken at local positions of the nanorod to judge the alignment of the corresponding regions. Notably, only the right part of the nanorod could be aligned to the electron beam, while the left region gradually tilted away from the zone axis as observed from the tilted diffraction spots in the FDs (TEM images of the first lines in Figure S7a-j). Upon tilting of the nanorod along the axial direction, the well-aligned region (indicated by the red box) gradually moved from the right to the left side (TEM images of the second lines in Figure S7a-j). 
View along $[110]_{\mathrm{cub}} /[11 \overline{2} 0]_{\text {hex }}$

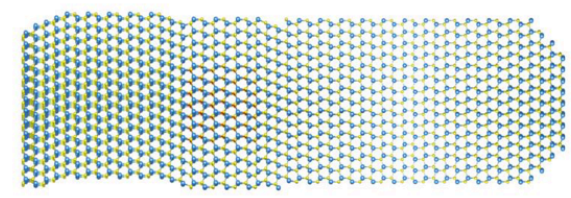

View along $[211]_{\mathrm{cub}} /[10 \overline{10}]_{\text {hex }}$

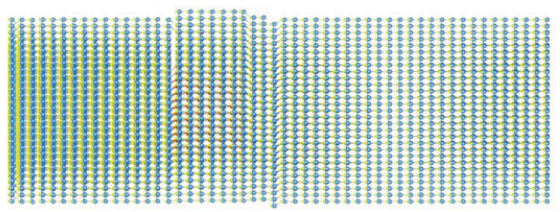

View along $[\overline{1} \overline{1} \overline{1}]_{\text {cub }} /[000 \overline{1}]_{\text {hex }}$

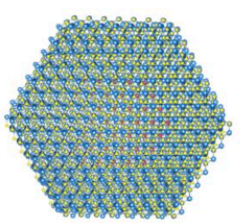

Figure S8. 3D models of the structure of the CCCN. Three images are viewed along $[110]_{\mathrm{cub}} /[11 \overline{2} 0]_{\mathrm{hex}}$, $[211]_{\mathrm{cub}} /[10 \overline{1} 0]_{\text {hex }}$ and $[111]_{\mathrm{cub}} /[0001]_{\text {hex }}$ directions.

A right-handed CCCN model was built to interpret the realistic helical structure shown in Figure 1c. The CdSe core with the diameter of $\sim 2.5 \mathrm{~nm}$ was enclosed in $\mathrm{CdS}$ shell. As a rotational structure derived from tilting operation of $\mathrm{CCCN}$, a structural model with continue twisting of $\sim 0.11 \% \mathrm{~nm}$ was applied on $3 \mathrm{D}$ model of CCCN, which features $\sim 1.87^{\circ}$ rotation for a length of $17 \mathrm{~nm}$. 

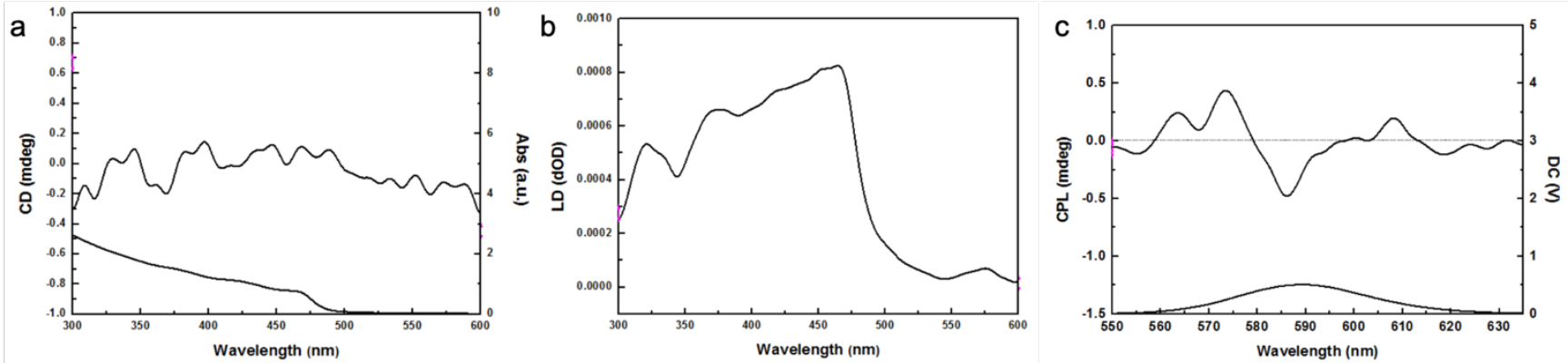

Figure S9. CD and UV-Vis spectra (a), LD spectra (b) and CPL and PL spectra (c) of CCCNs in toluene solution. 


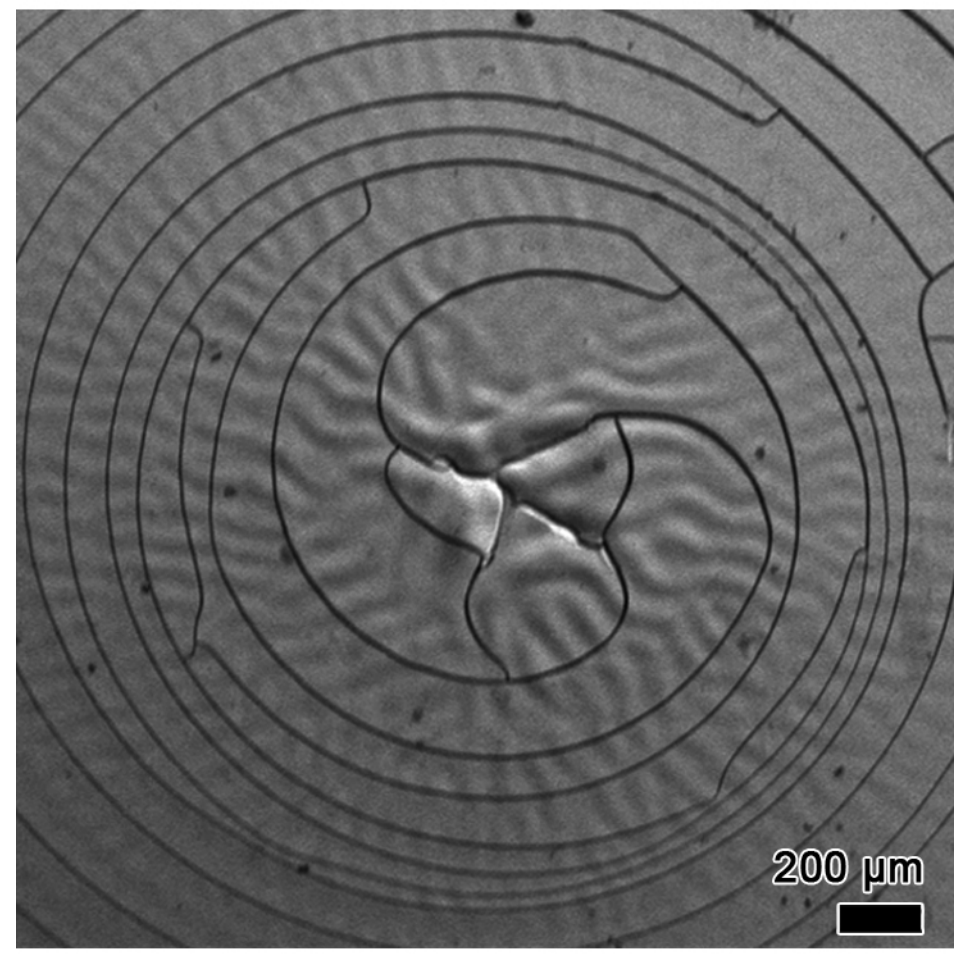

Figure S10. Top view SEM image of CNF.

The low magnification SEM image of CNF clearly showed the ridges on the surface, which would be due to the surface stress imbalance created by helical planks of the spherulite structure in the films. 


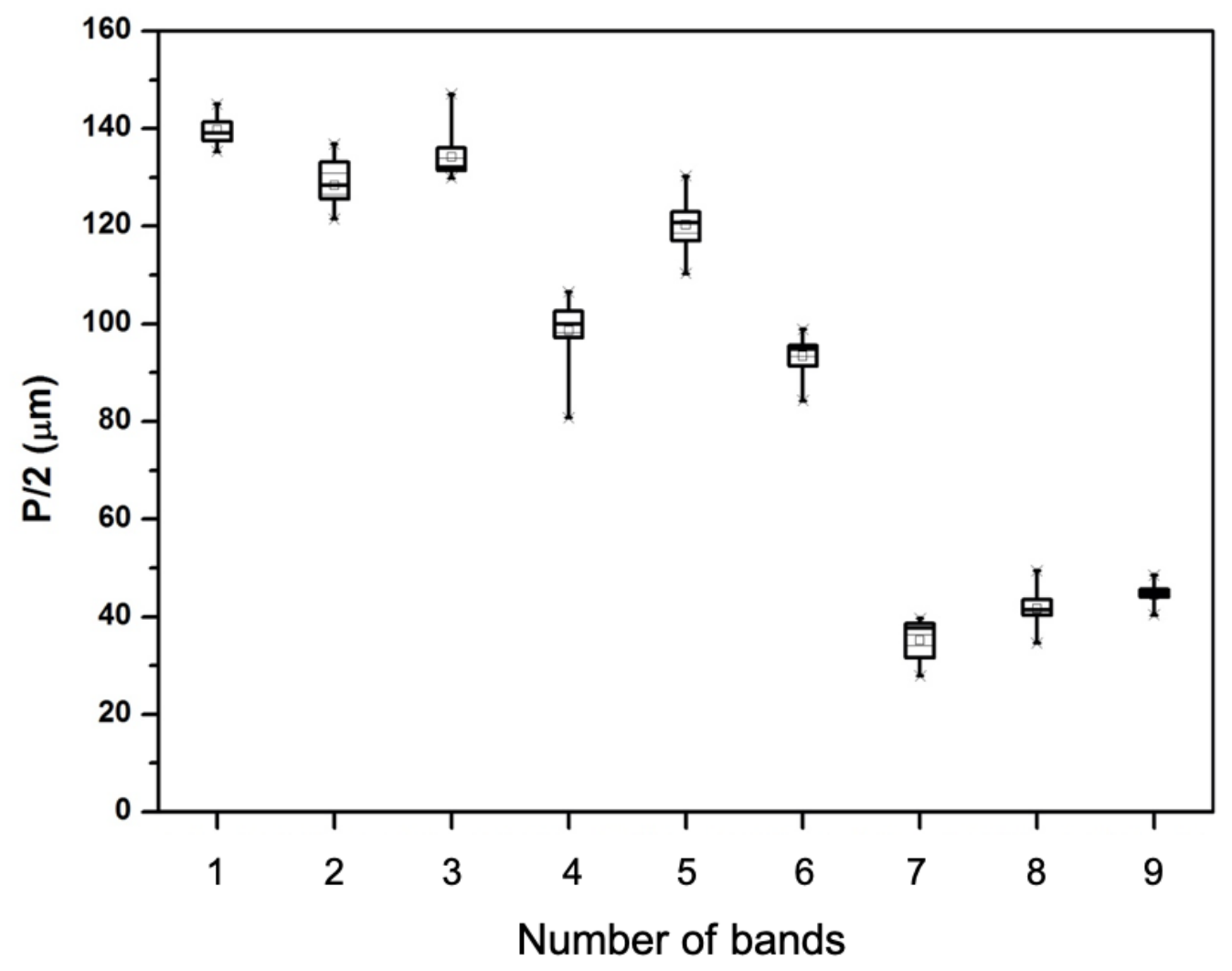

Figure S11. Half pitch length statistics for the tertiary chirality consisted of helical planks.

The experiment result indicates the pitch derived from the image cover a broad range from 30 140 micron, because evaporation rate was inhomogeneous at different positions during the volatilization of toluene. 

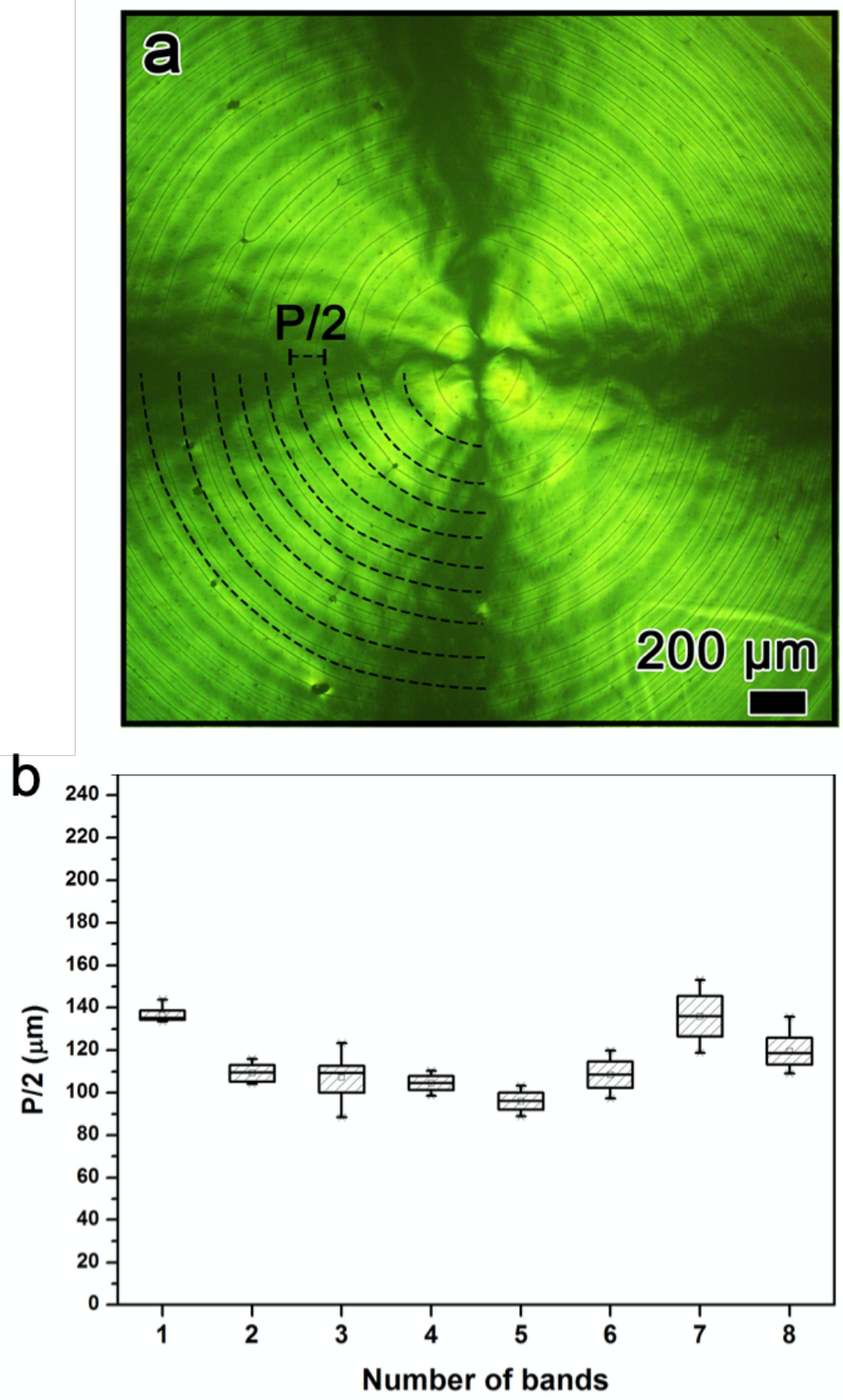

Figure S12. CNF prepared through control of evaporation process. (a) POM images under cross-polarized light. (b) Half pitch length statistics for the tertiary chirality consisted of helical planks.

The experiment result indicates the pitch derived from the image cover a narrow range from 100 140 micron, because evaporation rate was controlled during the volatilization of toluene. 

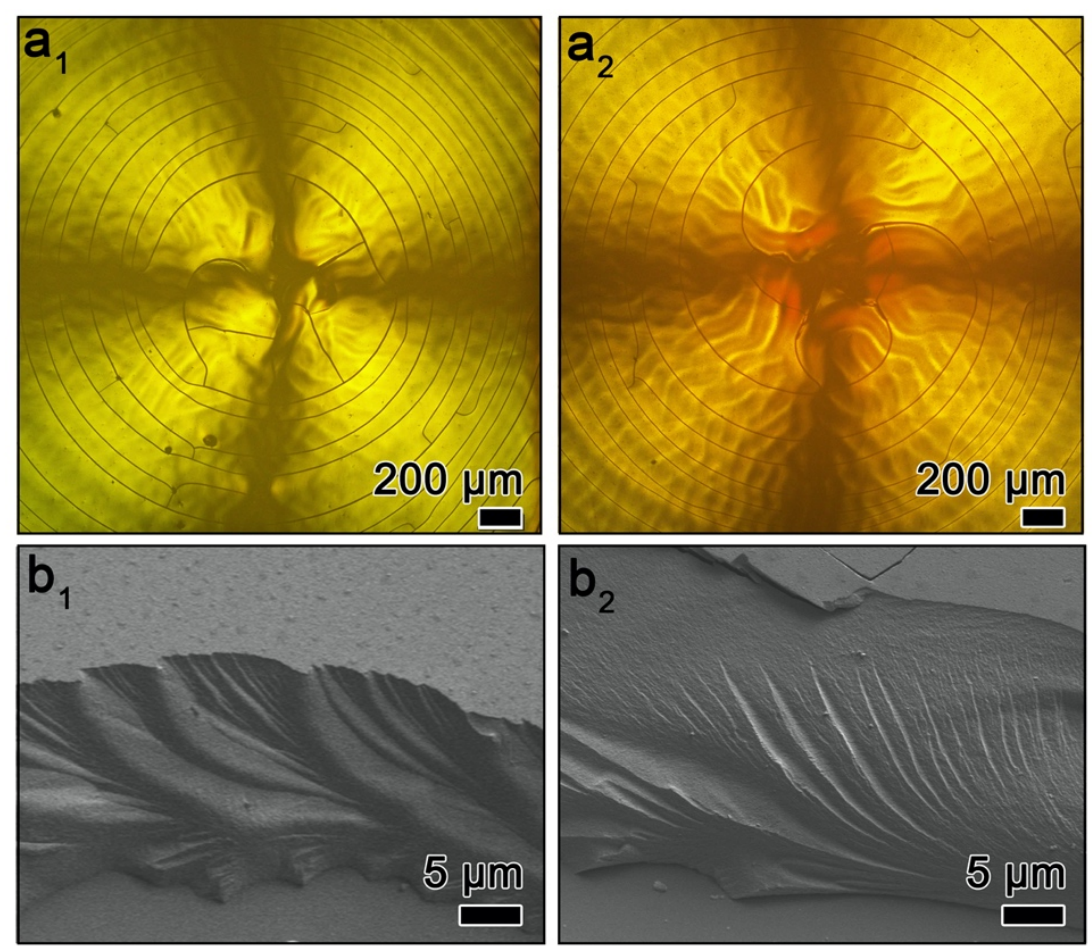

Figure S13. The POM images and SEM images of CNFs. $\left(\mathrm{a}_{1}, \mathrm{a}_{2}\right)$ The CNF with thickness of $\sim 17$ and $\sim 24 \mu \mathrm{m}$, respectively. $\left(b_{1}, b_{2}\right)$ SEM images of CNF in $a_{1}$ and $a_{2}$, respectively.

The color of the spherulites under cross-polarized light indicates its birefringence $(\Delta \mathrm{n}=\Gamma / \mathrm{t})^{2}$. The relationship between the birefringence color of a crystal is determined by the thickness of a crystal ( $\mathrm{t}$ ) and the optical retardation $(\Gamma)$. The retardation or "path difference" represents the difference between the fast ray and the slow ray produced along the path of light in a crystal and is therefore dependent on $\mathrm{t}$ and $\Delta \mathrm{n}$. A birefringence table, such as Michel-Levy chart, gives the relation between the Newton color and the thickness at a determined birefringence. Thus, with the increasing thickness of films, while the optical retardation lies in second order or third order, the colors of the spherulites under cross-polarized light is red-shift. (This is Figure of Michel-Levy color chart has been found in www.zeiss.com/microscopy.) 


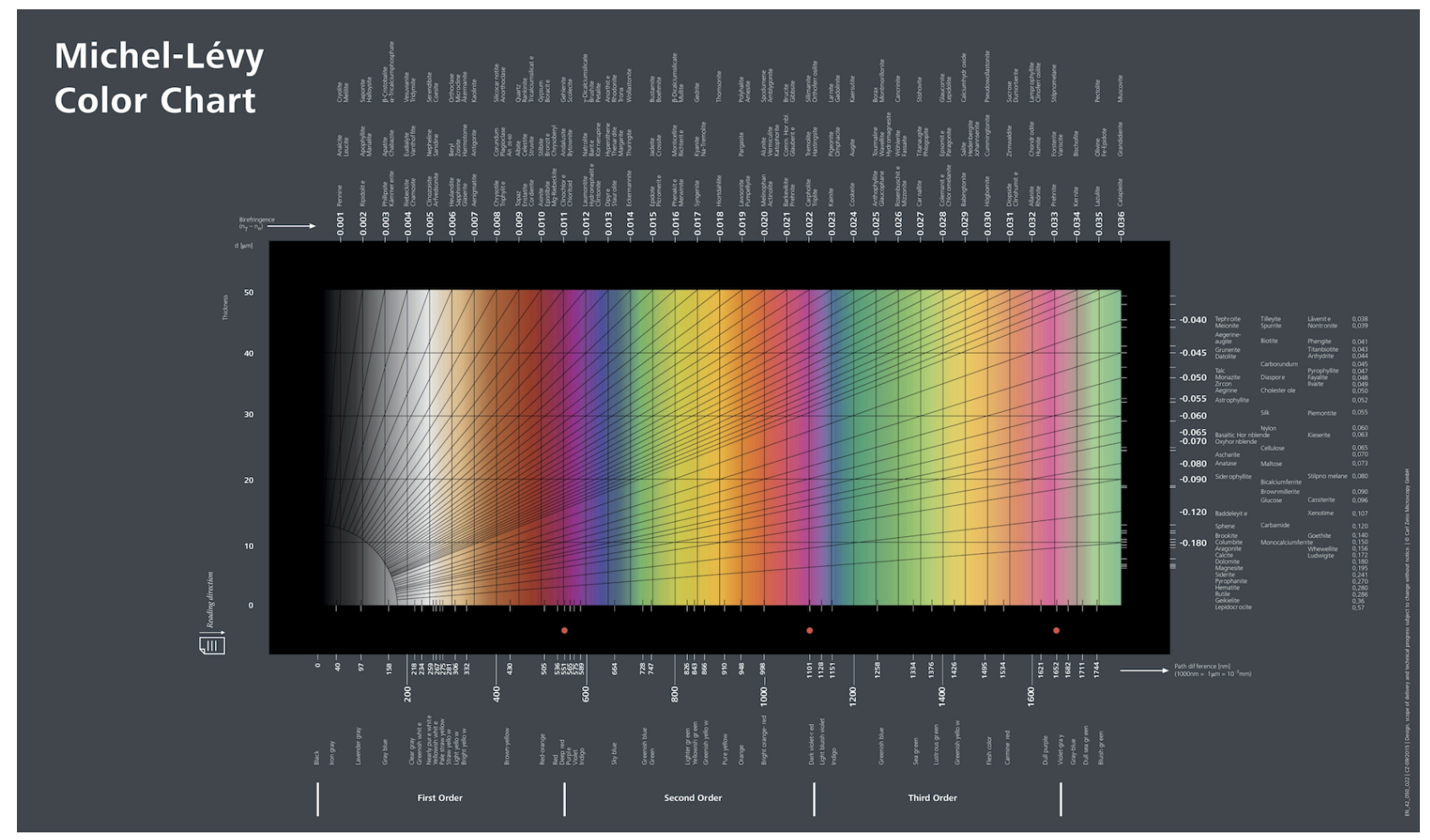



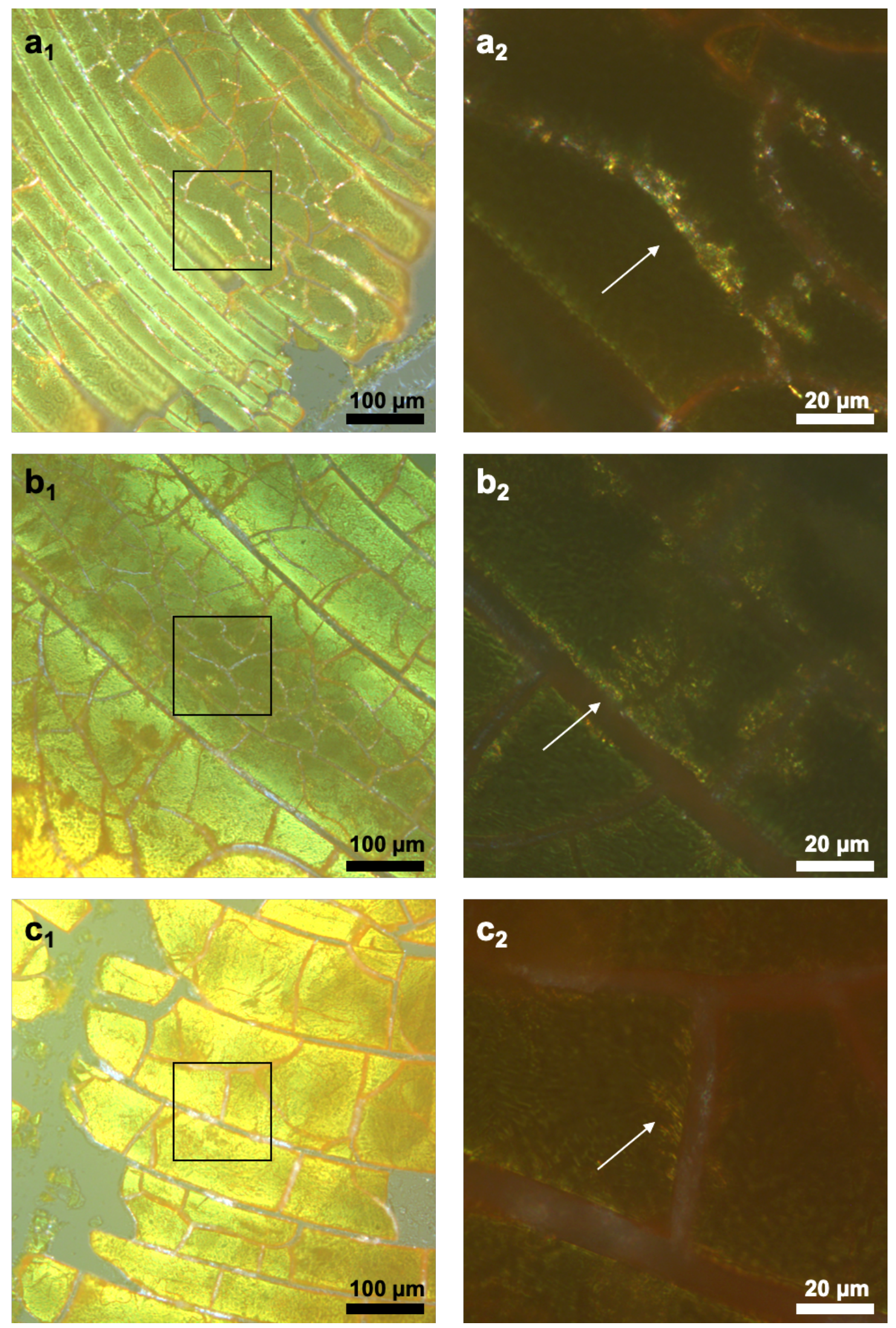

Figure S14. POM images of CNFs with thickness of with $8 \mu \mathrm{m}\left(\mathrm{a}_{1}, \mathrm{a}_{2}\right), 17 \mu \mathrm{m}\left(\mathrm{b}_{1}, \mathrm{~b}_{2}\right)$ and $24 \mu \mathrm{m}\left(\mathrm{c}_{1}, \mathrm{c}_{2}\right)$, respectively. 
High magnification POM images were added to further explicit the chiral nematic-like structure. Besides, the oxygen plasma cleaning was carried out to eliminate the optical influence of cholic acid and other organic molecules. The POM images show the films maintained circular bands after plasma but with some cracks (Figure S14a $a_{1}, b_{1}$ and $c_{1}$ ), the finger print texture of chiral nematic-like phase can be distinguished under high magnification for CNFs with different thickness (Figure $\mathrm{S}_{14} \mathrm{a}_{2}, \mathrm{~b}_{2}$ and $\mathrm{c}_{2}$, pointed by white arrows), indicating the films assembled in the form of chiral nematic-like structure. 

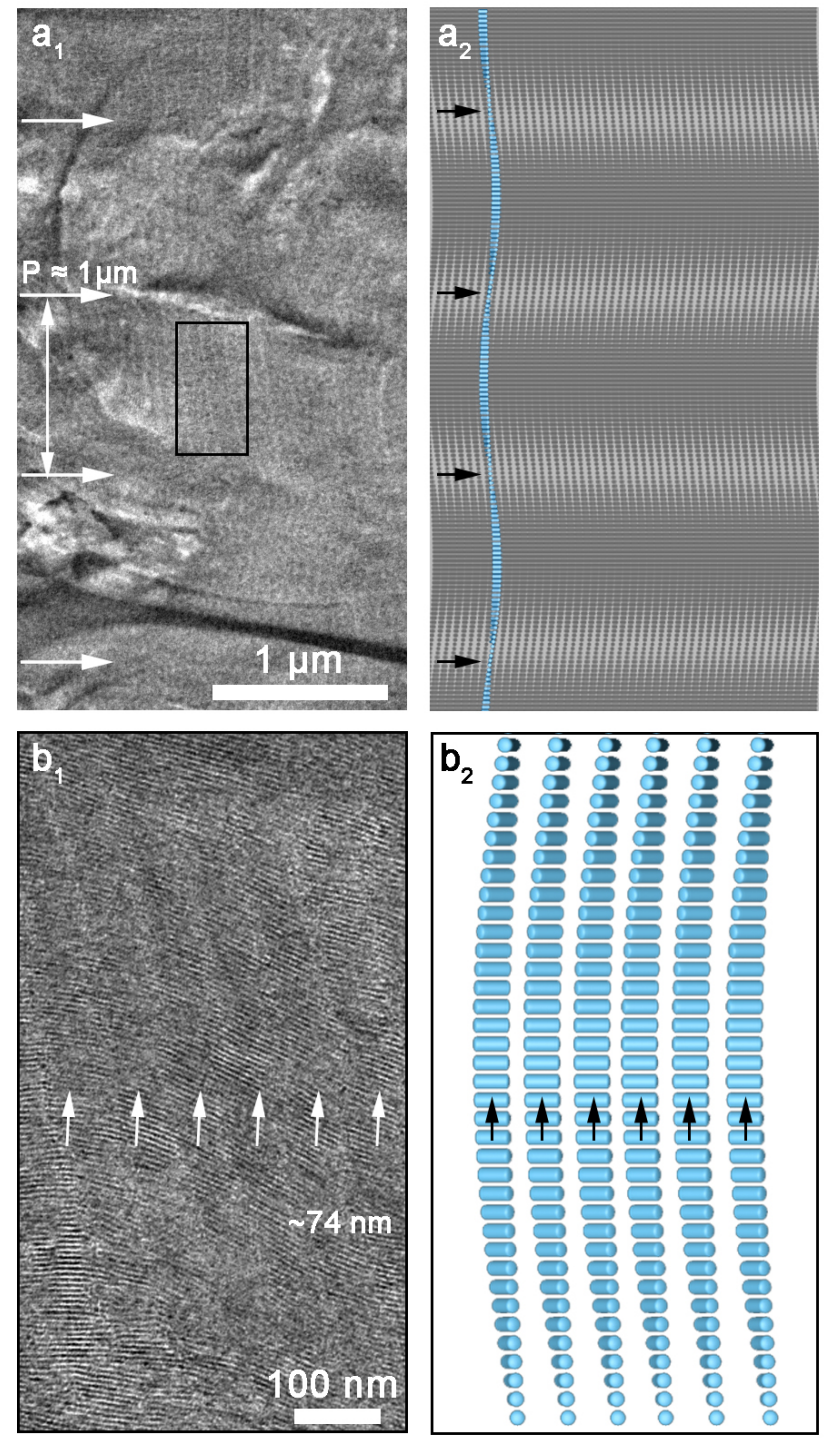

\section{Figure S15. The cross-sectional TEM images ( $a_{1}$ and $\left.b_{1}\right)$ of CNF and cross-sectional TEM models of the} chiral nematic-like structure ( $a_{2}$ and $\left.b_{2}\right)$.

The cross-sectional TEM images of the CNFs were taken from thin slices of the sample embedded in epoxy resin. As shown in Figure S15, the secondary chirality level is consistent with that in Figure 3. It was observed that the contrast corresponding to the parallel arrangement and the perpendicular arrangement of the nanorods is alternatively repeated at $\sim 1 \mu \mathrm{m}$ intervals, as marked by arrows (Figure $\mathrm{S} 15 \mathrm{a}_{1}$ ). The TEM models of the chiral nematic CCCN assembly (Figure S15 $\mathrm{a}_{2}$ ) was derived from the inset of Figure S15a 1 , which was in accordance with the TEM images and the half pitch length shown in Figure $3 b_{1}$. From high magnification in Figure $\mathrm{S} 15 b_{1}$, the chiral nematic CCCN assembly was composed of densely arranged sheets with a distance of $\sim 74 \mathrm{~nm}$, corresponding to the structural feature

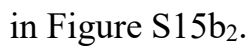



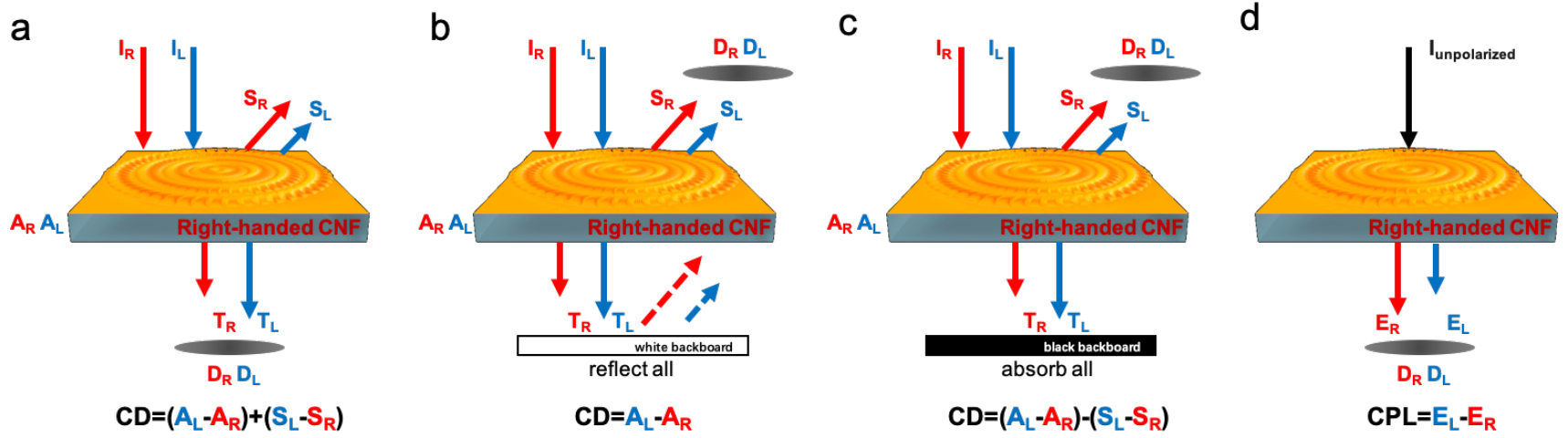

Figure S16. Schematic illustration of optical activities. (a) Interaction between circular polarized light and CNFs under the circumstance of transmittance UV-Vis. (b, c) CD spectra diffuse reflectance modes with white and black. (d) Circular polarized luminescence spectra.

As shown in the schematic illustration (Figure S16a), the transmitted CD signals contains both absorption-based parts and scattering based parts $\left[C D=\left(A_{L}-A_{R}\right)+\left(S_{L}-S_{R}\right)\right]$. Therefore, we performed the diffuse-reflection $C D$ with white $\left(\mathrm{DRCD}_{\mathrm{w}}\right)$ and black $\left(\mathrm{DRCD}_{\mathrm{B}}\right)$ background test, which approximately show the solely AOA and both SOA and AOA, because almost all light would be reflected by white and absorbed by black background (Figure S16b and S16c). Additionally, the signals of SOA with white and black background are opposite because transmitted and reflected light were detected by the TCD and DRCD system, respectively. Besides, the CPL signals contains only the emission parts of tested materials (Figure S16d). 
a

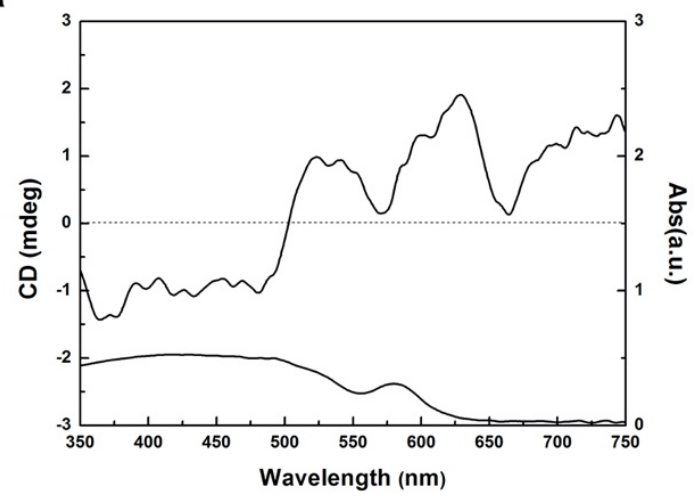

b

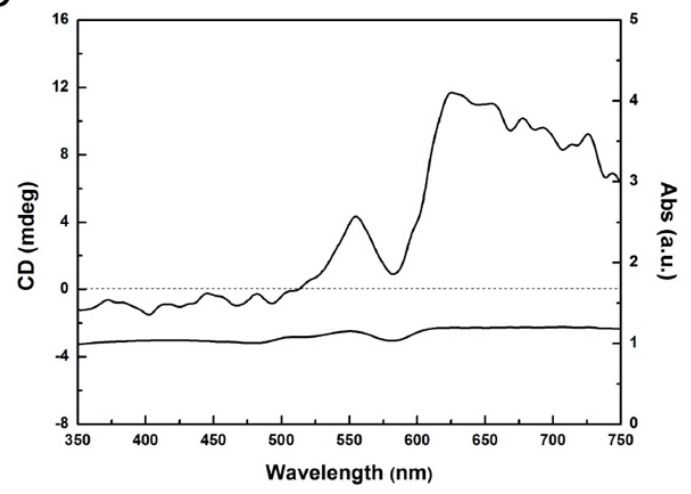

Figure S17. DRCD and UV-Vis diffuse reflection spectra of CNFs. (a) DRCD ${ }_{\mathrm{W}}$ and UV-Vis diffuse reflection spectra of CNFs. (b) DRCD $\mathrm{B}$ and UV-Vis diffuse reflection spectra of CNFs.

The peaks in $\mathrm{DRCD}_{\mathrm{W}}$ (Figure S17a) were generated all from ETOA. The peaks in $\mathrm{DRCD}_{\mathrm{B}}$ (Figure S17b) were generated through the combination of SOA and ETOA. The DRCDW of the CNF displayed positive peaks in the range of 500-750 nm. Meanwhile, the $\mathrm{DRCD}_{\mathrm{B}}$ of the CNF displayed positive peaks in the range of 500-750 $\mathrm{nm}$. However, the peaks of $\mathrm{DRCD}_{\mathrm{B}}$ in the range of $500-750 \mathrm{~nm}$ is positive contrast to TCD signals, which could be due to the stronger positive signals of SOA than AOA. Thus, it can be concluded that the negative signals of TCD (Figure 4a) in the region of $500 \mathrm{~nm}$ to $750 \mathrm{~nm}$ were generated from strong SOA and weak ETOA, because the SOA signals are opposite in the TCD and DRCD as illustrated in Figure S16. 


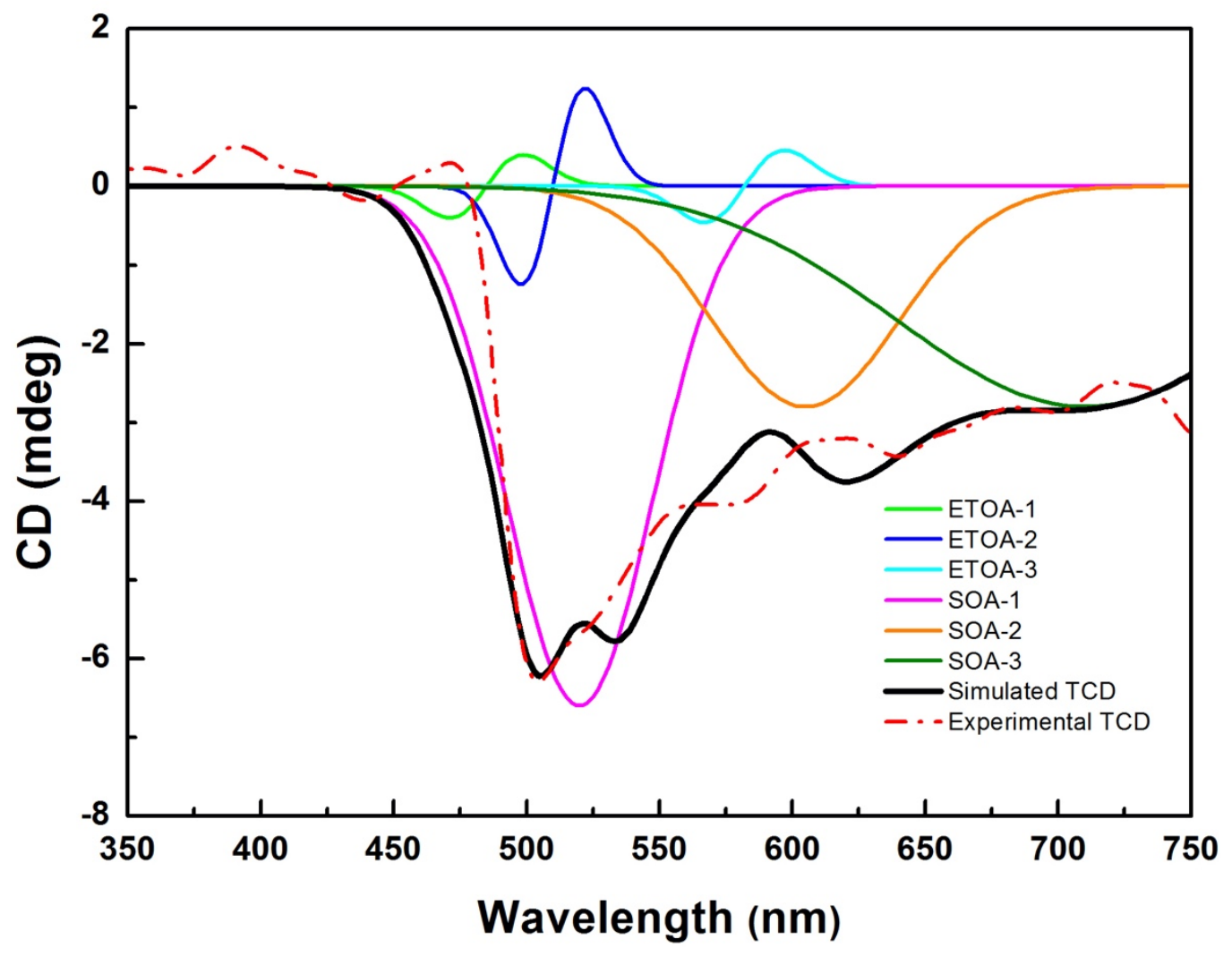

Figure S18. Schematic illustration of composition of TCD signal. Experimental TCD signal for the CNF is a combination of simulated ETOA signals and SOA signals.

TCD was analysed through simulating AOA and SOA. TCD signals of right-handed CNF were generated through the combination of both negative SOA and analogical positive AOA. It is known that a medium with right-handed structures prefers to reflect right-handed circularly polarized light and absorb left-handed circularly polarized light. The primary distorted crystal lattices formed helical nanorods is relatively too weak to be considered. Thus, the signals in the region from 500-750 nm resulted from hierarchically chiral assembly of CCCNs, which features positive electron transitions absorption-based split-type Cotton Effect centered at $485 \mathrm{~nm}$ (ETOA-1 in Figure S18), $510 \mathrm{~nm}$ (ETOA-2), $582 \mathrm{~nm}$ (ETOA-3); scattering based optical activity at $520 \mathrm{~nm}$ (SOA-1), $605 \mathrm{~nm}$ (SOA-2), and $710 \mathrm{~nm}$ (SOA-3). The three scattering peaks were generated by the primary, secondary, and tertiary level assembly structures in the CNF 

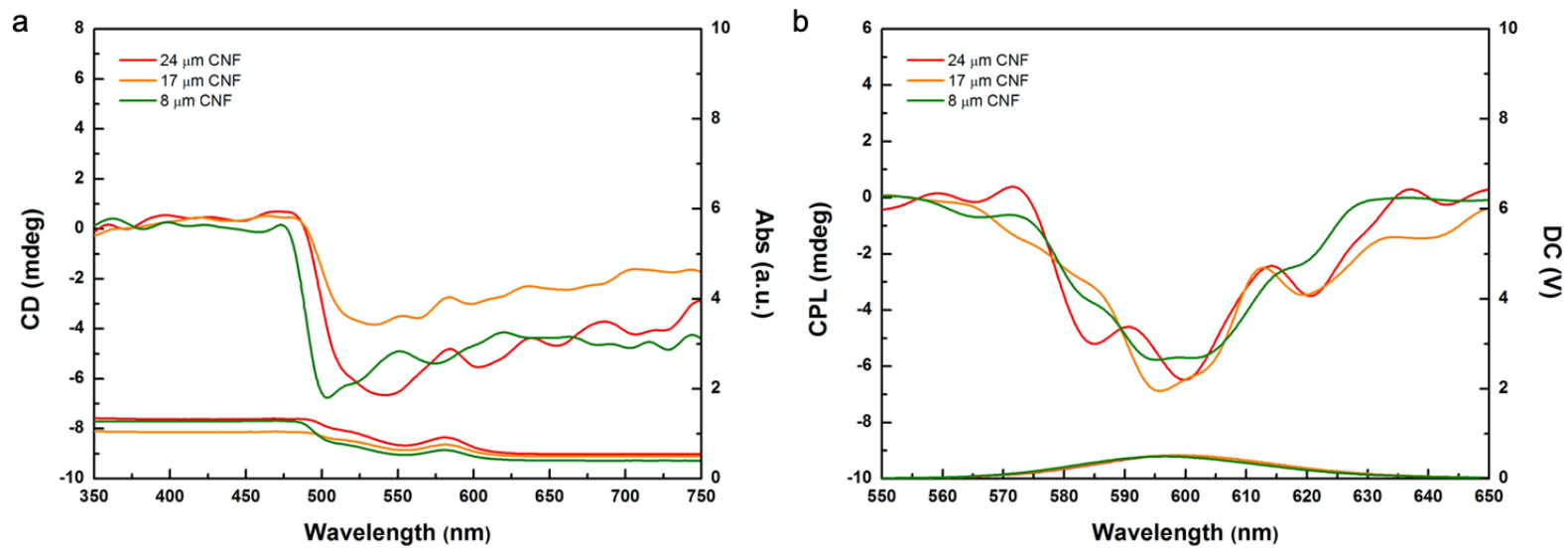

Figure S19. OAs of CNFs with thickness of $24 \mu \mathrm{m}, 17 \mu \mathrm{m}$ and $8 \mu \mathrm{m}$. (a) CD and UV-Vis spectra. (b) CPL and PL spectra.

As shown in Figure S19a, CNF with thickness of $24 \mu \mathrm{m}, 17 \mu \mathrm{m}$ and $8 \mu \mathrm{m}$ exhibit three absorption bands centred at $\sim 582, \sim 510$ and $\sim 485 \mathrm{~nm}$, corresponding to the excitonic transitions of CdS and CdSe. The TCD spectra of three CNFs show negative peaks in the range of $500-750 \mathrm{~nm}$, indicating that left-handed circularly polarized light preferentially transmitted to the films. As shown in Figure S19b, the CPL spectra of the CNFs show negative signals at $\sim 600 \mathrm{~nm}$ coinciding with the emission wavelength in the photoluminescence (PL) spectra, confirming the existence of right-handed chiral structures in all CNFs. 

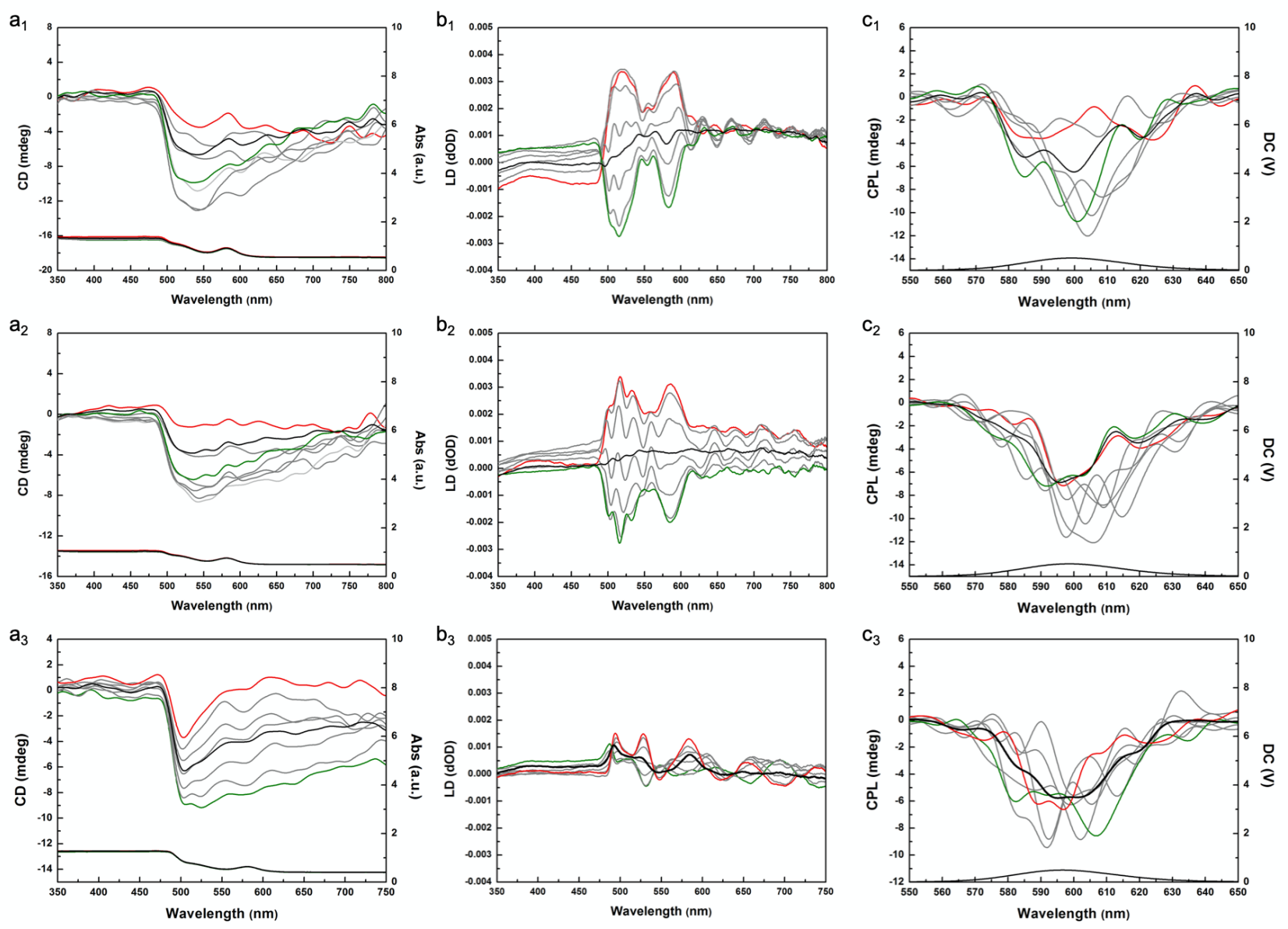

Figure S20. CD and UV-Vis spectra ( $\left(a_{1}, a_{2}\right.$ and $\left.a_{3}\right)$, LD spectra $\left(b_{1}, b_{2}\right.$ and $\left.b_{3}\right)$ and CPL and PL spectra $\left(c_{1}, c_{2}\right.$ and $\left.c_{3}\right)$ of CNFs with thickness of $24 \mu \mathrm{m}, 17 \mu \mathrm{m}$ and $8 \mu \mathrm{m}$, respectively.

According to the convention, the effect of linear dichroism (LD) on the CD and CPL was examined using the "rotation" CD spectra ${ }^{3}$. For the substrate with vertical stripes, LD is the largest when the parallel light is parallel to the vertical stripes (referred to red line), and the smallest when the parallel light is orthogonal to the vertical stripes (referred to green line). For the material with line dichroism, the sum of LD vector can be compared to the substrate with vertical stripes (referred to black line). Therefore, the $\mathrm{CD}$ spectrum corresponding to $\mathrm{LD}=0$ can be obtained by accumulation of the $\mathrm{CD}$ corresponding to the minimum value of $\mathrm{LD}$ and the maximum value of $\mathrm{LD}$. In the same way, it can be applied to CPL spectra. Thus, it can be concluded that the negative signals of TCD and CPL were obtained without the influence of LD. 

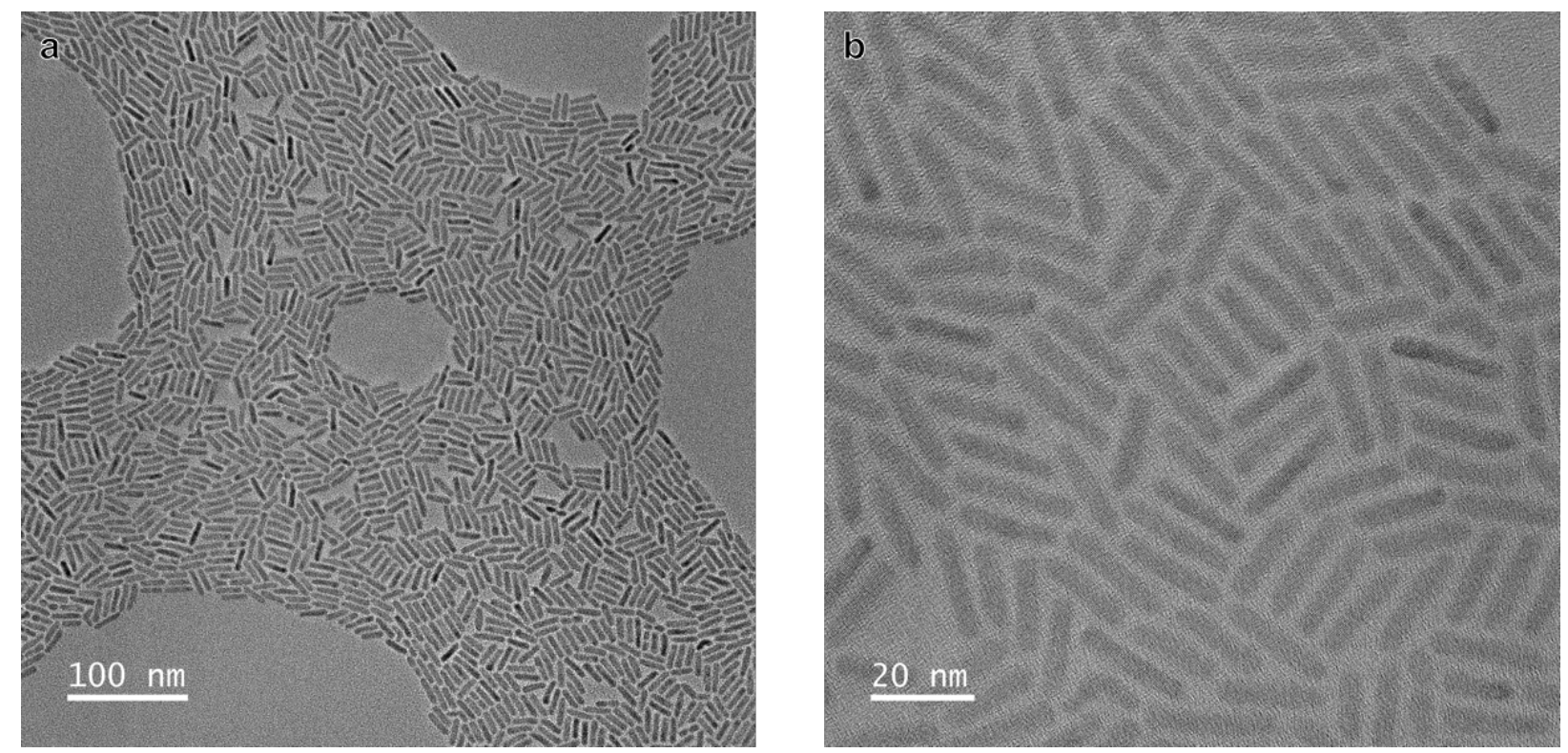

Figure S21. TEM images of CdSe/CdS nanorods without addition of cholic acid.

To illustrate the difference among CCCNs, achiral CdSe/CdS and achiral CdSe/CdS mixing with $\mathrm{CA}$, we synthesized achiral CdSe/CdS nanorods without addition of cholic acid. The TEM images is shown in Figure S21. 
a

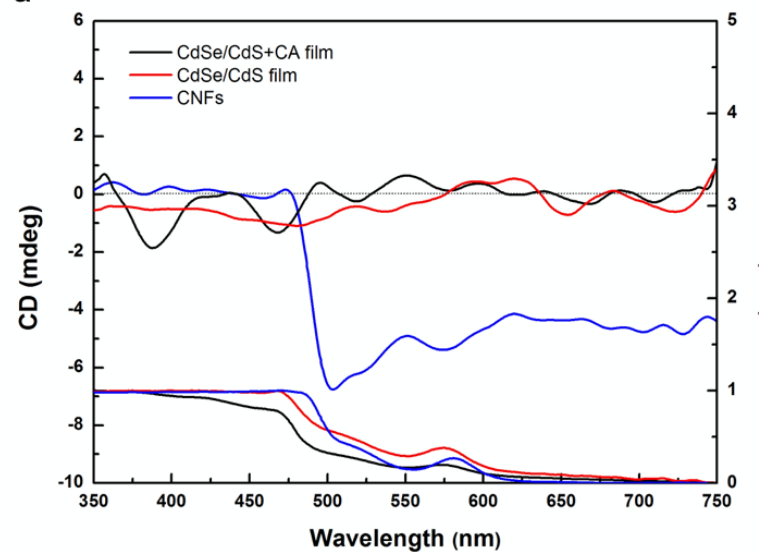

b

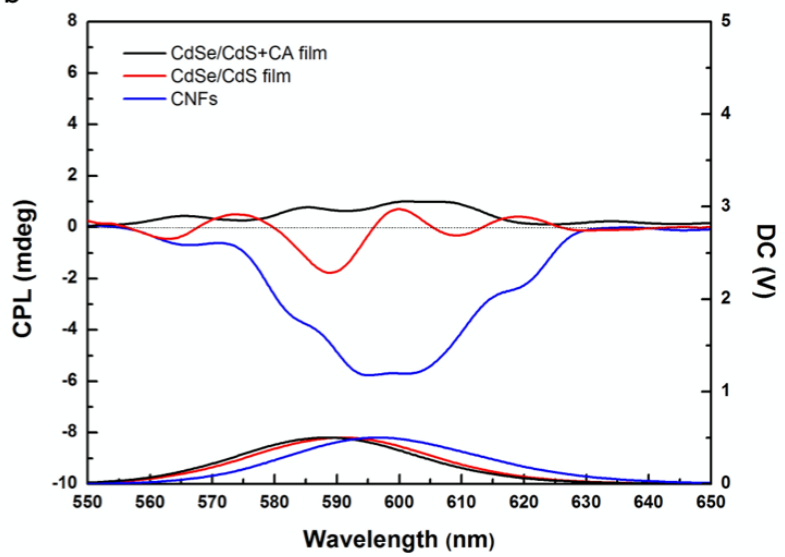

Figure S22. OAs for the films assembled with mixing CA with achiral CdSe/CdS nanorods, achiral CdSe/CdS and CCCNs. (a) CD and UV-Vis spectra. (b) CPL and PL spectra.

(i) Films prepared by mixing CA with achiral nanorods: Firstly, the CA was dissolved in a toluene solution. Because the solubility of CA in toluene is not good, the prepared solution is toluene saturated solution of CA $\left(20{ }^{\circ} \mathrm{C}\right)$. Secondly, the achiral CdSe/CdS nanorods were dispersed in a saturated CA (toluene) solution to form a nanorod dispersion of $\sim 30 \mathrm{mg} / \mathrm{mL}$ (almost a saturated colloidal solution). $20 \mu \mathrm{L}$ dispersion solution of nanorod mixed with CA was dropped on the $0.5 \mathrm{~cm} \times 0.5 \mathrm{~cm}$ substrate to volatilize and self-assemble into films.

As shown in Figure S22a, the CD spectra of film assembled by mixing CA with achiral CdSe/CdS shows weak signal in the absorption bands corresponding to $350-500 \mathrm{~nm}$ probably because of the formation of nanorod assembly via the induction of CA molecules leading to weak interaction selectivity between the medium and circularly polarized light. The CPL spectra show that the CA with achiral CdSe/CdS film have almost silent circularly polarized luminescence signals, indicating that the chirality is weak for assembly structure via directly mixing CA with achiral $\mathrm{CdSe} / \mathrm{CdS}$. The results show that CA is not the reason for the formation of chiral structure of CNFs.

(ii) Films prepared by achiral nanorods without CA: The experiment was explored as follow: the achiral $\mathrm{CdSe} / \mathrm{CdS}$ nanorods were dispersed in toluene solution to form a nanorod dispersion of $\sim 30 \mathrm{mg} / \mathrm{mL}$. $20 \mu \mathrm{L}$ dispersion solution of nanorod was dropped on the $0.5 \mathrm{~cm} \times 0.5 \mathrm{~cm}$ substrate to volatilize and self-assemble into films.

As shown in Figure S22b, the CD spectra of film assembled by achiral CdSe/CdS also shows silent signal in the absorption bands corresponding to 350-650 $\mathrm{nm}$. The CPL exhibits almost silent circularly polarized luminescence signals of achiral CdSe/CdS film indicating the racemate state or weak interaction selectivity between the $\mathrm{CdSe} / \mathrm{CdS}$ film and circularly polarized light. The results show that the achiral CdSe/CdS nanorods synthesized without CA mentioned above could not form chiral structure of CNFs. 

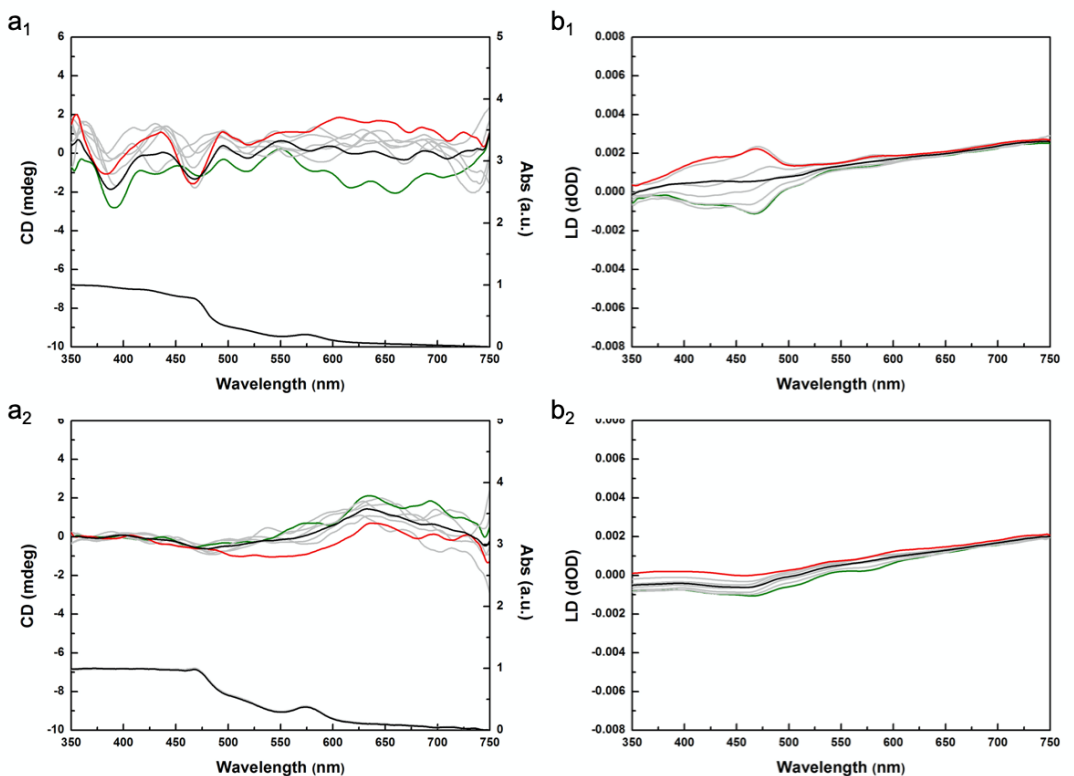

$\mathrm{b}_{2}$

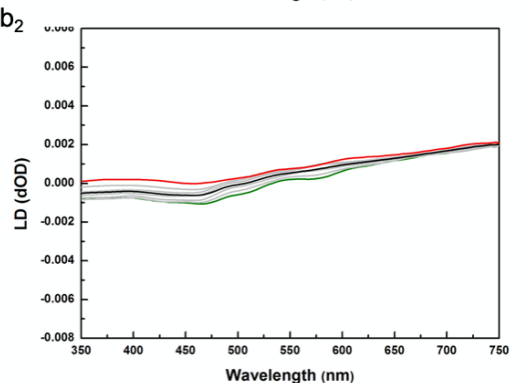

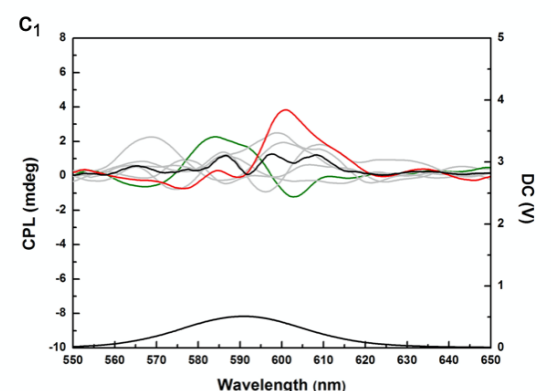

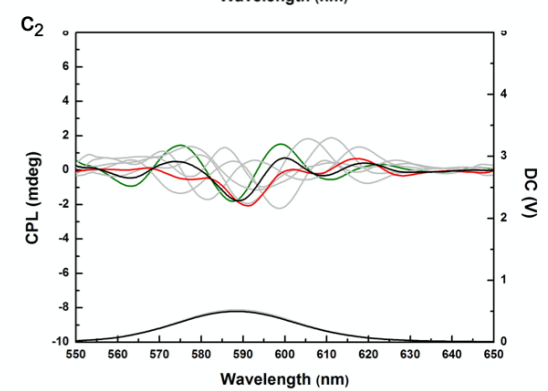

Figure S23. CD and UV-Vis spectra ( $\left(a_{1}\right.$ and $\left.a_{2}\right)$, LD spectra $\left(b_{1}\right.$ and $\left.b_{2}\right)$ and CPL and PL spectra ( $c_{1}$ and $\left.c_{2}\right)$ for the films assembled by mixing CA with achiral CdSe/CdS nanorods and achiral CdSe/CdS.

The detailed elimination procedure of linear dichroism (LD) in the CD, LD, and CPL spectra for the films assembled by mixing CA with achiral CdSe/CdS nanorods and achiral CdSe/CdS is shown in Figure S23. 


\section{References}

1. Yu, W. W.; Qu, L.; Guo; Wenzhuo; Peng, X., Experimental Determination of the Extinction Coefficient of CdTe, CdSe, and CdS Nanocrystals. Chemistry of Materials 2003, 15 (14), 2854-2860.

2. Beaufort, L.; Barbarin, N.; Gally, Y., Optical measurements to determine the thickness of calcite crystals and the mass of thin carbonate particles such as coccoliths. Nature Protocols 2014, 9 (3), 633-42.

3. Yeom, B.; Zhang, H.; Zhang, H.; Park, J. I.; Kim, K.; Govorov, A. O.; Kotov, N. A., Chiral Plasmonic Nanostructures on Achiral Nanopillars. Nano Letters 2013, 13 (11), 5277-5283. 